\title{
Numerical Experiment for Dipole-Dipole Interaction in Electro-Magnetism with Help of a Regular Tetrahedron
}

\author{
Panagis G. Papadopoulos ${ }^{1}$, Panos D. Kiousis ${ }^{2}$, Christos G. Karayannis ${ }^{3}$ \\ ${ }^{1}$ Laboratory of Structural Engineering, Department of Civil Engineering, Aristotle University of Thessaloniki, Thessaloniki, Greece \\ ${ }^{2}$ Laboratory of Structural and Geotechnical Engineering, Department of Civil and Environmental Engineering, Colorado School of \\ Mines, Golden, Colorado, USA \\ ${ }^{3}$ Laboratory of Concrete Structures, Department of Civil Engineering, Democritus University of Thrace, Komotini, Greece \\ Email: panaggpapad@yahoo.gr
}

How to cite this paper: Papadopoulos, P.G., Kiousis, P.D. and Karayannis, C.G. (2019) Numerical Experiment for Dipole-Dipole Interaction in Electro-Magnetism with Help of a Regular Tetrahedron. Open Journal of Physical Chemistry, 9, 13-32.

https://doi.org/10.4236/ojpc.2019.91002

Received: January 26, 2019

Accepted: February 25, 2019

Published: February 28, 2019

Copyright $\odot 2019$ by author(s) and Scientific Research Publishing Inc. This work is licensed under the Creative Commons Attribution International License (CC BY 4.0).

http://creativecommons.org/licenses/by/4.0/

\begin{abstract}
Aim of this work is to try to explain, on a Rational basis, some equations of Electro-Magnetism, which are based on Experimental data. Any Electric Field can produce a Field of many small Electric Dipoles, continuously distributed in space. In a region, where the Electric Field is constant, in direction and magnitude, all the small Dipoles are parallel to the Electric Field, and are represented by a single, long, parallel to them, fixed in space, Electric Dipole, which is here called Compass. An Alternating current, in a straight Conductor, is studied, by a simple, short computer program, for step-by-step nonlinear dynamic analysis. It is found that, only an Alternating current, not a direct current, can produce an Electric Dipole, in a straight Conductor. The two above Dipoles (Compass-Conductor) are assumed with equal lengths $\ell$, lying on two skew lines, perpendicular to each other, at a distance $\ell / \sqrt{2}$, thus forming, by their four ends, a Regular Tetrahedron, with side length $\ell$. Repulsion, between Like Charges, obeys the simple Coulomb Electro-Static law. Whereas Interaction (Attraction or Repulsion), between Unlike Charges, obeys a more accurate Lennard-Jones law. The analysis of Dipole-Dipole (Compass-Conductor) Interaction is performed by hand calculator. The only out-of-balance forces, in the regular Tetrahedron, acting on the Rigid Conductor, are the so-called magnetic forces. Their direction is found, in a simple Rational way, with help of Regular Tetrahedron, without recoursing to a "right-hand-rule". The proposed model is applied to 1) The force acting on an Electric Charge moving in a magnetic field. 2) The force acting on a Current carrying straight Conductor, due to a magnetic field. 3) The magnetic fields created around a Current carrying straight Conductor. In these applica-
\end{abstract}


tions, proposed model gives reasonable results. Particularly, in third application, results, obtained by proposed model, are found in satisfactory approximation with corresponding ones, obtained by an empirical formula, based on relevant Experimental observations of H.-C. Oersted and A.-M. Ampère. So, the reliability of proposed model is checked. Position and direction of magnetic field vector coincide with those of a corresponding fixed Compass of a constant Electric Field. Main point of present work is that, without introducing the concept of a magnetic field vector, by combining field of dipoles, produced by an electric field, with dipole of an alternating current carrying conductor, the magnetic forces can be determined.

\section{Keywords}

Electric Field, Electric Dipole, Compass, Conductor, Alternating Current, Step-by-Step Nonlinear Dynamic Algorithm, Dipole-Dipole Interaction, Regular Tetrahedron, Coulomb Electro-Static Law, Lennard-Jones Law, Magnetic Field Vector, Magnetic Forces, Oersted-Ampère Rule

\section{Introduction}

Some fundamental equations of Electro-Magnetism are based on Experimental data, e.g. those concerning the force acting on an Electric Charge moving in a magnetic field, as well as the magnetic fields created around a Current carrying straight Conductor [1] [2]. Aim of present work is to try to explain the above equations on a Rational basis.

Any Electric Field can produce a Field of many small Electric Dipoles, continuously distributed in space. In a region where the Electric Field is constant, in direction and magnitude, all the small Dipoles are parallel to the Electric Field, and are represented by a single, long, fixed in space, Electric Dipole, which is here called Compass. This is in accordance to the concept of FEM (Finite Elements Method) that a continuous medium has to be Discretized to a Discontinuous one, so that a Computation becomes possible [3] [4].

An Alternating Current, in a straight Conductor, is studied by a simple and short computer program, for Step-by-Step Nonlinear Dynamic Analysis [5] [6] [7]. It is found that only an Alternating current, not a direct current, can produce an Electric Dipole, in a straight Conductor, in agreement with relevant Experimental observations by Michael Faraday and Nikola Tesla [8] [9].

The above two Electric Dipoles, the fixed Compass of a constant Electric Field and the Alternating current carrying straight Conductor, are assumed with equal lengths $\ell$, lying on two skew lines, perpendicular to each other, at a distance $\ell / \sqrt{2}$, thus forming, by their four ends, a Regular Tetrahedron with side length $\ell[10]$.

Repulsion, between a couple of Like Electric Charges, obeys the simple Coulomb Electro-Static law [11]. Whereas, Interaction (Attraction or Repulsion), 
between a couple of Unlike Electric Charges, obeys a more accurate Lennard-Jones law [12].

The proposed model is applied to 1) The force acting on an Electric Charge moving in a magnetic field. 2) The force acting on a Current carrying straight Conductor, due to a magnetic field. 3) The magnetic fields created around a Current carrying straight Conductor [1] [2], and gives reasonable results.

Particularly in the third application, results, obtained by the proposed model, are found in satisfactory approximation with corresponding ones, obtained by an empirical formula based on relevant Experimental observations by H.-C. Oersted and A.-M. Ampère [13] [14]. So, the reliability of proposed model is checked.

By non-introducing the concept of a magnetic field vector and by considering a dipole-dipole (Compass-Conductor) Interaction, with help of a Regular Tetrahedron, the calculations, in practical applications, can be based on a Rational basis and significantly Simplified.

\section{Proposed Model}

\subsection{Electric Field Producing Field of Electric Dipoles}

Any Electric Field can produce a Field of many small, continuously distributed in space, Electric Dipoles. In a region where the value (direction and magnitude) of the Electric Field can be approximately considered constant, all these many small Electric Dipoles are approximately oriented parallel to the Electric Field, as shown in Figure 1(a), and are represented here by a single, long, parallel to them, fixed in space, Electric Dipole, as shown in Figure 1(b), which, from this point on, is here called "Compass".

The above simulation is in accordance with the concept of FEM (Finite Elements Method) that a continuous medium has to be Discretized to a Discontinuous one, so that a Computation becomes possible [3] [4].

At the two ends of resultant Compass, of Figure 1(b), two opposite Electric Charges $\pm q$ appear. By considering static equilibrium of each one of these two Charges, along axis of Compass, under action of external Electric Field, on one hand, and action of internal Electro-Static Coulomb Attraction, on the other, is obtained:

$$
F=E q-k q^{2} / \ell^{2}=0,
$$

where $E$ magnitude of Electric Field, $\ell$ length of resultant Compass and $k=8.9875 \times 10^{9} \mathrm{~N} \cdot \mathrm{m}^{2} / \mathrm{C}^{2}$ is the Coulomb Electro-Static constant.

So, the above equation results to

$$
q=E \ell^{2} / k,
$$

that is, the absolute value $\mathrm{q}$ of the two opposite Electric Charges, at ends of fixed resultant Compass, is proportional to magnitude $E$ of Electric Field and to square $\ell^{2}$ of Compass length, and inversely proportional to Coulomb Electro-Static constant $\mathrm{k}$. 
(a)

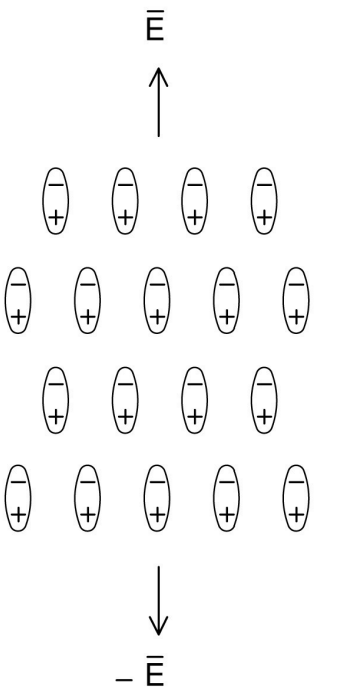

(b)

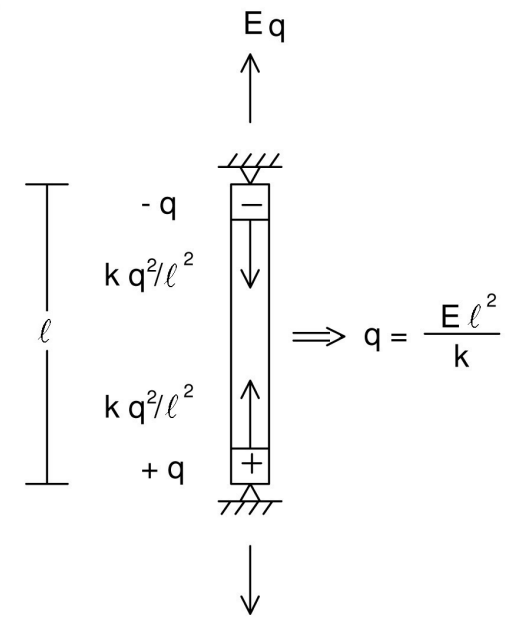

Figure 1. In a region of space, where the value (direction and magnitude) of Electric Field is approximately constant. (a) The many small Electric Dipoles are approximately parallel to the Electric Field. (b) The many small Dipoles are represented by a single, long, parallel to them, fixed in space, Electric Dipole, called here "Compass".

\subsection{Alternating Current in a Straight Conductor}

A straight Conductor, with cross-section area A and length $\ell$, is considered, as shown in Figure 2(a).

By some EMF (electromotive force), e.g. a battery, a small initial drift speed $v_{0}$, to the right, is given, to a Free Electrons Cloud, in the Conductor. So, this Cloud is initially displaced to right. At some instant, the displacement of the Cloud to right is $+x$, as shown in Figure 2(a), where $x \ll \ell$. In this figure, the $x$ is shown exaggerated, that is, much larger than in reality, for demonstration.

At left end of Conductor, the Free Electrons have been removed and Positive Ions of Conductor atoms remain. So, a Positive Electric Charge is formed:

$$
+q=+n \text { Axe, }
$$

where $n$ number of Free Electrons per unit volume of Conductor, and $e=1.6 \times 10^{-19} \mathrm{C}$ Electric Charge of one Electron

At right end of Conductor, Electrons are accumulated along an equal distance $+x$. So, an excess of Negative Electric Charge $-q=-n$ Axe is formed at right end of Conductor.

According to Coulomb electrostatic law, the two opposite electric charges $\pm q$, at ends of Conductor, are attracted by a force $F=+k q^{2} / \ell^{2}$.

The above force $F$ is applied on the Free Electrons Cloud, directed to left, as shown in Figure 2(b), and gives to it acceleration to left.

$$
\gamma=F / M
$$

where $M=n A \ell m_{e}$ is total mass of Free Electrons Cloud and $m_{e}=9.11 \times 10^{-31} \mathrm{~kg}$ mass of one Electron.

The above acceleration $\gamma$, directed to left, first, is retarding the movement of 
(a)

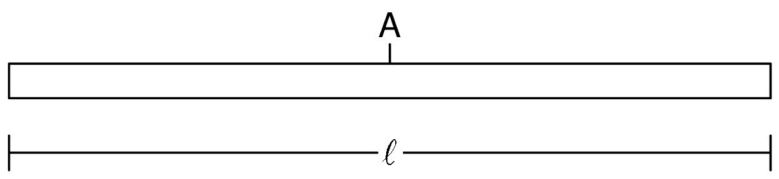

(b)

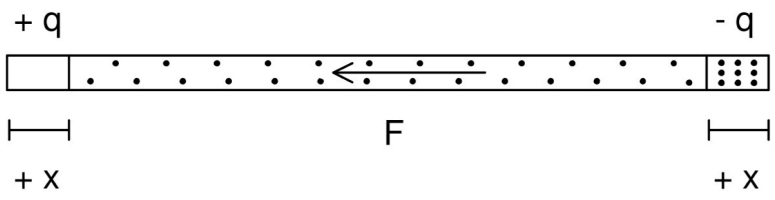

(c)

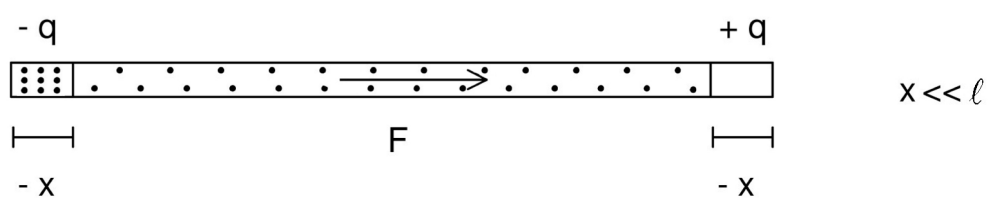

Figure 2. (a) A straight Conductor with cross-section area $A$ and length $\ell$. (b) Free Electrons Cloud is displaced to right, by a distance $+x$, where $x \ll \ell$. A positive electric charge $+q$ is formed at left end of the Conductor and a negative charge $-q$ at right end. The Coulomb electrostatic attractive force $F$, between the two opposite charges $\pm q$, at ends of Conductor, is acting on Free Electrons Cloud, directed to left. (c) The Free Electrons Cloud is displaced to left by a distance $-x$, where $x \ll \ell$. Now, a positive electric charge $+q$ is formed at right end of Conductor and a negative charge $-q$ at left end. The Coulomb electrostatic attractive force $F$, between the two opposite charges $\pm q$, at ends of Conductor, is acting on Free Electrons Cloud, directed, now, to right.

Free Electrons Cloud to right, then movement is directed to left, and, after some time instant, the Polarity of the Conductor is reversed, as shown in Figure 2(c).

Now, distances, along which electric charges are formed, at two ends of Conductor, are noted as negative $-x$, where again $x \ll \ell$. And positive electric charge $+q$ is formed at right end of Conductor, whereas negative electric charge $-q$ is formed at left end of Conductor.

In above movement of Free Electrons Cloud to left, force $F$, from Coulomb attraction, between the two opposite electric charges, at ends of Conductor, is acting on Free Electrons Cloud, directed now to right, and gives to it acceleration $\gamma=F / M$ to right.

This acceleration $\gamma$ of Free Electrons Cloud to right, first, retards its movement to left, then movement is directed to right, and, after some time instant, polarity of Conductor is reversed and becomes again as in Figure 2(b).

The whole above procedure is repeated, so Free Electrons Cloud oscillates along the Conductor, which is an Alternating current.

\subsection{Study of Alternating Current by a Step-by-Step Nonlinear Dynamic Program}

The Alternating Current in a straight Conductor, that is, the oscillation of Free Electrons Cloud, along the Conductor, can be studied by a step-by-step nonli- 
near dynamic algorithm [5] [6] [7]. Here, the simple algorithm of trapezoidal rule is chosen, combined with predictor-corrector technique, with one prediction and two corrections per step, noted as $P E(C E)^{2}$, where $P$ prediction, $E$ evaluation and $C$ correction [7].

The time steplength $\Delta t$ of algorithm is determined by trials. First, an arbitrary value of $\Delta t$ is chosen. If the solution of problem diverges, $\Delta t$ is gradually reduced, until a convergence of solution is achieved.

The criteria for stability and accuracy of algorithm, in determination of time steplength $\Delta t$, are the following [7]:

Stability criterion, $\omega \Delta t<2.0 \mathrm{rad} \rightarrow \Delta t<T / \pi$,

Accuracy criterion, $\omega \Delta t<0.5 \mathrm{rad} \rightarrow \Delta t<T / 4 \pi$,

where $T$ period of oscillation of Free Electrons Cloud. This period $T$ will be clearly shown, in time-histories of problem variables, in numerical example of next Section 2.4.

The proposed algorithm is described by the flow-chart of Figure 3 .

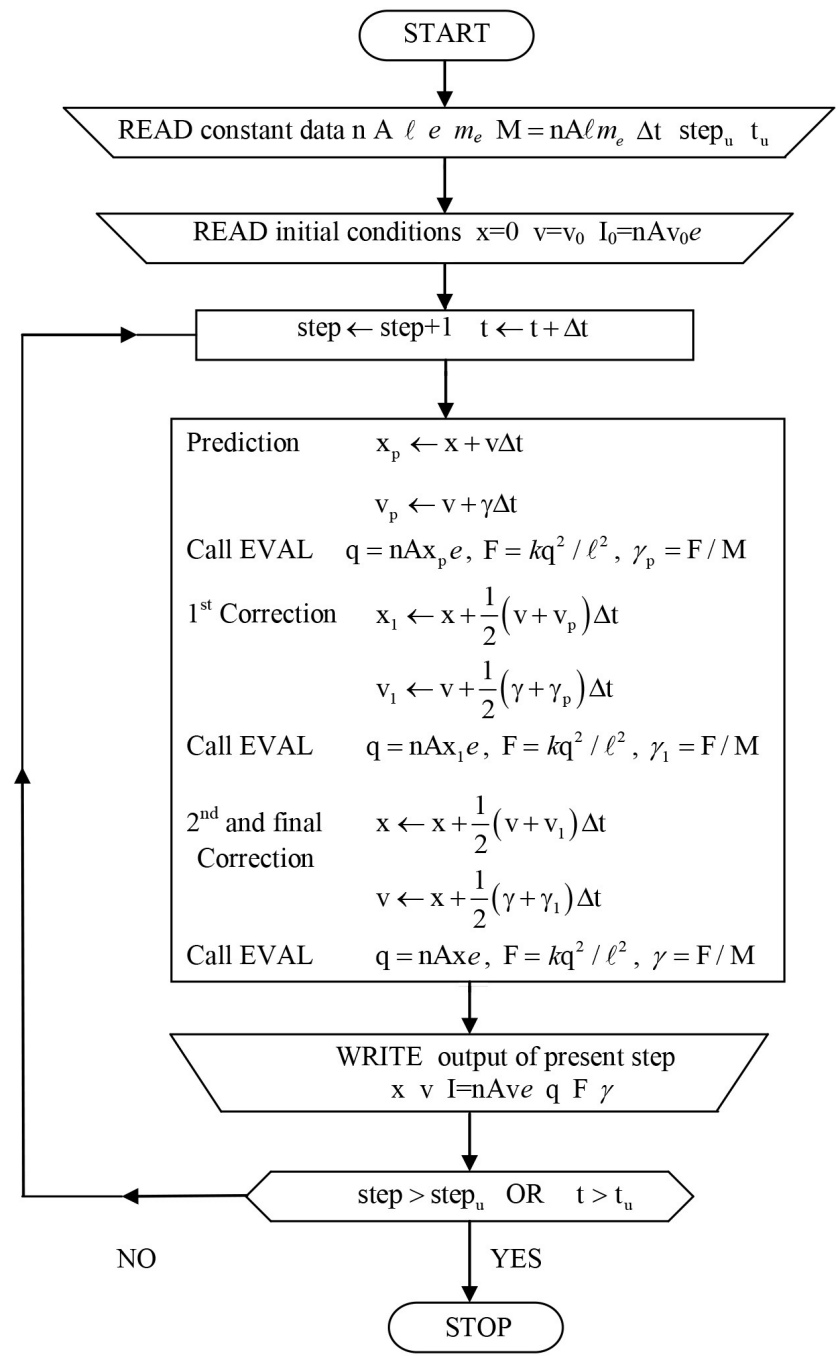

Figure 3. Flow-chart of proposed algorithm, for step-by-step nonlinear dynamic analysis of Alternating Current in a straight Conductor. 
First the constant data of problem are read: $n$ number of Free Electrons per unit volume of Conductor, $A, \ell$ cross-section area and length of Conductor, $e$, $m_{e}$ electric charge and mass of one electron. From these data, the total mass of Free Electrons Cloud is determined $M=n A \ell m_{e}$.

Also, in the constant data, are read: time step-length $\Delta t$ of algorithm, as well as chosen upper bounds step $_{u}, t_{u}$, for number of algorithm steps and time, above which the algorithm have to be interrupted.

Then, the initial values of state variables are read: $x=0$ zero initial displacement, and $v=v_{0}$ initial drift speed of Free Electrons Cloud. From $v_{0}$, the initial value of Current $I_{0}=n A v_{0} e$ is determined.

Within each step of the algorithm, one prediction and two corrections, by trapezoidal rule, for the values of state variables $X, V$, are performed. After prediction and each one of two corrections, the subroutine EVAL is called, which evaluates the electric charges $q= \pm n A x e$, at the two ends of Conductor, the Coulomb attraction force between them $F=+k q^{2} / \ell^{2}$, which acts on the Free Electrons Cloud, and the acceleration of Cloud $\gamma=F / M$.

At the end of every step of algorithm, output data are written: step (number of algorithm step), $t$ time, $x, v$ state variables, that is, displacement and drift speed of Free Electrons Cloud, $I=n A v e$ Current, $q=n A x e$ electric charge at ends of Conductor, $F=k q^{2} / \ell^{2}$ attractive force between the two opposite charges $\pm q$ at ends of Conductor, which acts on Free Electrons Cloud, and $\gamma$ acceleration of Cloud.

Then, the algorithm continues with next step.

When step or time t exceeds its chosen upper bound, the algorithm is interrupted.

\subsection{Numerical Example of an Alternating Current}

A Conductor made of Copper is considered. With the assumption that every atom of the Conductor offers one Free Electron, according to Example 17.2. Drift speed of electrons, pages 593 - 595 of [1], the number of Free Electrons, per unit volume of Conductor, results $n=8.46 \times 10^{28}$ electrons $/ \mathrm{m}^{3}$. A cross-section area $A=3.0 \times 10^{-6} \mathrm{~m}^{2}$ and a length $\ell=1.0 \mathrm{~m}$ are assumed for the Conductor. The electric charge of one electron is $e=1.6 \times 10^{-19} \mathrm{C}$. The mass of one electron is $m_{e}=9.11 \times 10^{-31} \mathrm{~kg}$, so the total mass of Free Electrons Cloud is only

$$
\begin{aligned}
M & =n A \ell m_{e} \\
& =8.46 \times 10^{28} \text { electrons } / \mathrm{m}^{3} \times 3.0 \times 10^{-6} \mathrm{~m}^{2} \times 1.0 \mathrm{~m} \times 9.11 \times 10^{-31} \mathrm{~kg} / \text { electron } \\
& =0.2312 \mathrm{mgr}
\end{aligned}
$$

The above constant data are first read, in the input of proposed algorithm, described by the flow-chart of Figure 3, of previous Section 2.3.

The time steplength $\Delta t$ of the algorithm is determined by trials. The period $T$ of oscillation of Free Electrons Cloud, that is, of Alternating Current, as will be shown in the output, in the time-histories of present variables problem, at the end of present section, in Figure 4, is: 
(a)

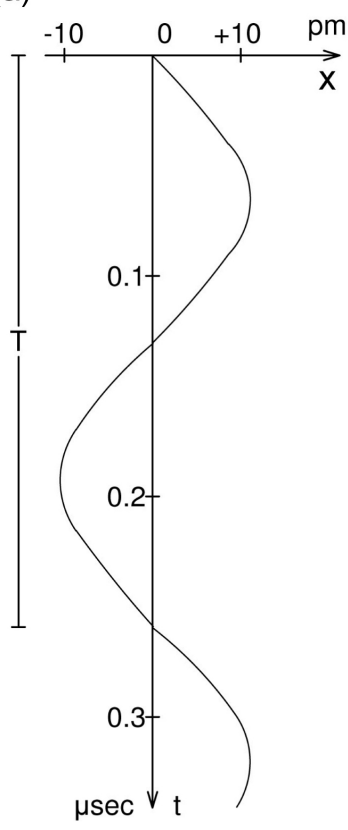

(d)

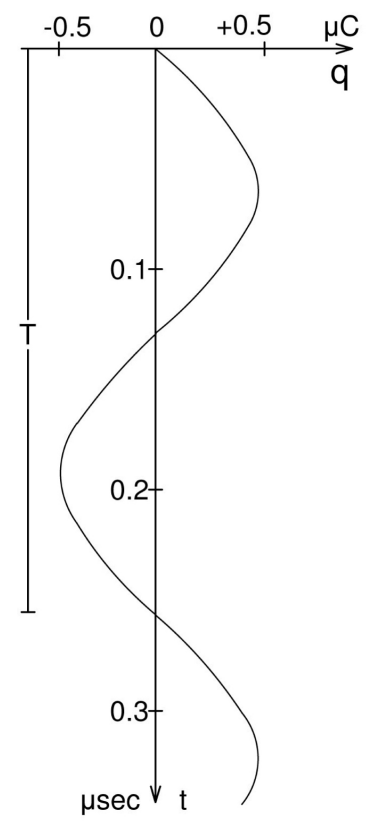

(b)

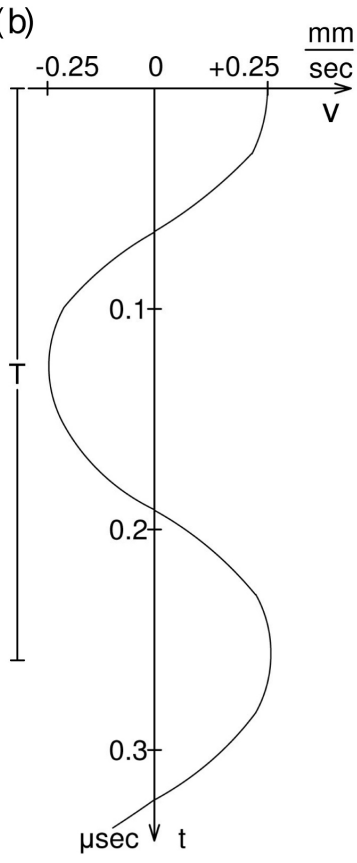

(e)

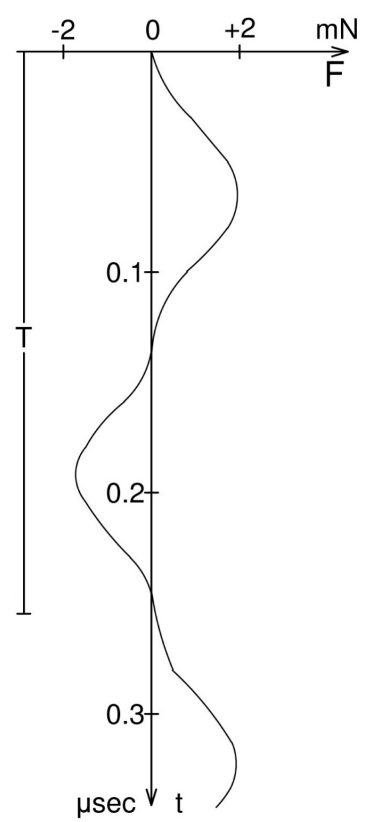

(c)

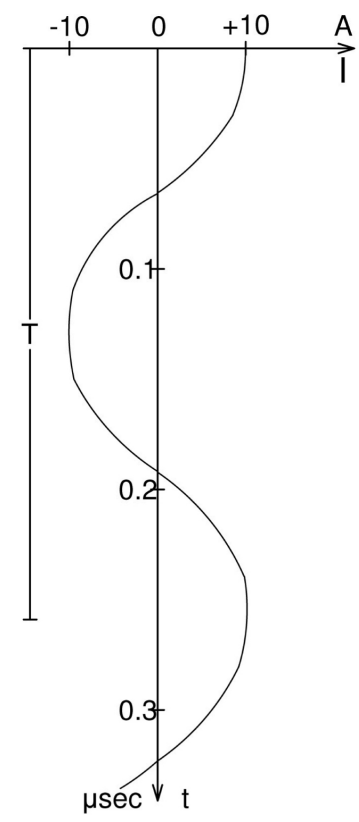

(f)

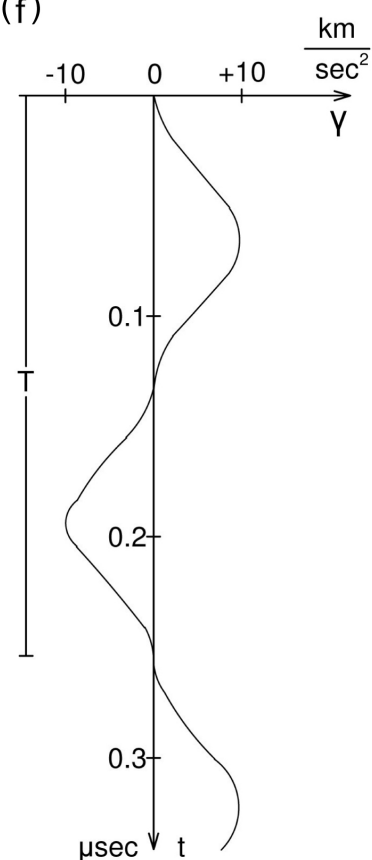

Figure 4. For numerical example of Section 2.4, on Alternating Current in a straight Conductor, output of time-histories of main variables of problem. In all diagrams, time $t$ is measured in $\mu \mathrm{sec}=10^{-6} \mathrm{sec}$ and period of oscillation is $T=0.26 \mu \mathrm{sec}=0.26 \times 10^{-6} \mathrm{sec}$. In the six time-histories, the problem variables and their units are the following: (a) Displacement $x$ of Free Electrons Cloud in $\mathrm{pm}=10^{-12} \mathrm{~m}$. (b) Drift speed $v$ of Free Electrons Cloud in $\mathrm{mm} / \mathrm{sec}=10^{-3} \mathrm{~m} / \mathrm{sec}$. (c) Current $I$ in A $=\mathrm{C} / \mathrm{sec}$. (d) Electric Charge $q$ at two ends of Conductor in $\mu \mathrm{C}=10^{-6} \mathrm{C}$. (e) Attractive force $F$ in $\mathrm{mN}=10^{-3} \mathrm{~N}$, between opposite Charges $\pm q$ at the ends of Conductor, acting on Free Electrons Cloud. (f) Acceleration $\gamma$ in $\mathrm{km} / \mathrm{sec}^{2}=10^{3} \mathrm{~m} / \mathrm{sec}^{2}$ of Free Electrons Cloud. 


$$
T=0.26 \times 10^{-6} \mathrm{sec}=0.26 \mu \mathrm{sec} .
$$

So, for accuracy of the algorithm, the time steplength $\Delta t$ have to satisfy the criterion

$$
\omega \Delta t<0.5 \mathrm{rad} \rightarrow \Delta t<\mathrm{T} / 4 \pi=0.26 \mu \mathrm{sec} / 12.57=0.02068 \mu \mathrm{sec} \approx 0.02 \mu \mathrm{sec} .
$$

For more accuracy $\Delta t=0.01 \mu \mathrm{sec}=10^{-8} \mathrm{sec}$ is chosen, that is, 26 steps per period $T$ of oscillation. And the upper bounds $\operatorname{step}_{u}=52$ and $t_{u}=0.52 \mu$ sec are set, for studying two periods of oscillation.

The initial conditions of state variables are zero displacement $x=0$ and drift speed $v=v_{0}=2.46257 \times 10^{-4} \mathrm{~m} / \mathrm{sec}=0.246257 \mathrm{~mm} / \mathrm{sec}$. So, the corresponding initial current is

$$
\begin{aligned}
I_{0}= & n A v_{0} e \\
= & 8.46 \times 10^{28} \text { electrons } / \mathrm{m}^{3} \times 3.0 \times 10^{-6} \mathrm{~m}^{2} \times 2.46257 \times 10^{-4} \mathrm{~m} / \mathrm{sec} \\
& \times 1.6 \times 10^{-19} C / \text { electron } \\
= & 10.0 \mathrm{C} / \mathrm{sec}=10.0 \mathrm{~A}
\end{aligned}
$$

With the above constant input data and initial conditions, and with steplength $\Delta t=0.01 \mu \mathrm{sec}$, the step-by-step nonlinear dynamic algorithm, described in previous Section 2.3 and by flow-chart of Figure 3, has run and gave the time-histories of the variables of present problem, shown in Figure 4. The time is measured in $\mu \mathrm{sec}=10^{-6} \mathrm{sec}$ and the period is $T=0.26 \mu \mathrm{sec}$ for time-histories of all variables.

The variables of the problem, whose time-histories are presented in Figure 4, are the following with their units: (a) Displacement $x$ of Free Electrons Cloud in $\mathrm{pm}=10^{-12} \mathrm{~m}$. (b) Drift speed $v$ of Free Electrons Cloud in $\mathrm{mm} / \mathrm{sec}=10^{-3} \mathrm{~m} / \mathrm{sec}$. (c) Current $\mathrm{I}$ in $\mathrm{A}=\mathrm{C} / \mathrm{sec} . \mathrm{d}$. Electric charge $q$ at the ends of Conductor in $\mu \mathrm{C}=10^{-6} \mathrm{C}$. (e) Attractive force $\mathrm{F}$ in $\mathrm{mN}=10^{-3} \mathrm{~N}$, between opposite charges at the ends of Conductor, acting on Free Electrons Cloud. (f) Acceleration $\gamma$ in $\mathrm{km} / \mathrm{sec}^{2}=10^{3} \mathrm{~m} / \mathrm{sec}^{2}$ of the Cloud.

It is observed, in the present numerical example, on Alternating Current in a straight Conductor, that, only an Alternating Current, not a direct current, can produce an Electric Dipole, in a straight Conductor, in agreement with Experimental observations by Michael Faraday and Nikola Tesla [8] [9].

\subsection{Dipole-Dipole (Compass-Conductor) Interaction with Help of a Regular Tetrahedron}

The two previously mentioned Electric Dipoles, that is, the fixed Compass, in a region of constant Electric Field (Section 2.1), and the Alternating Current carrying straight Conductor (Sections 2.2 up to 2.4) are considered, with equal lengths $\ell$, lying on two skew lines, perpendicular to each other, at a distance $\ell / \sqrt{2}$, thus forming, by their four ends, a Regular Tetrahedron, with side length $\ell$, as shown in the 3D representation of Figure 5, and in the three Projections of Figure 6 (Plan on plane $x y$. Elevation on plane $y z$. Side view on plane $x z$ ).

The analysis of Dipole-Dipole (Compass-Conductor) Interaction, with help of above Regular Tetrahedron, can be performed by a hand calculator. 


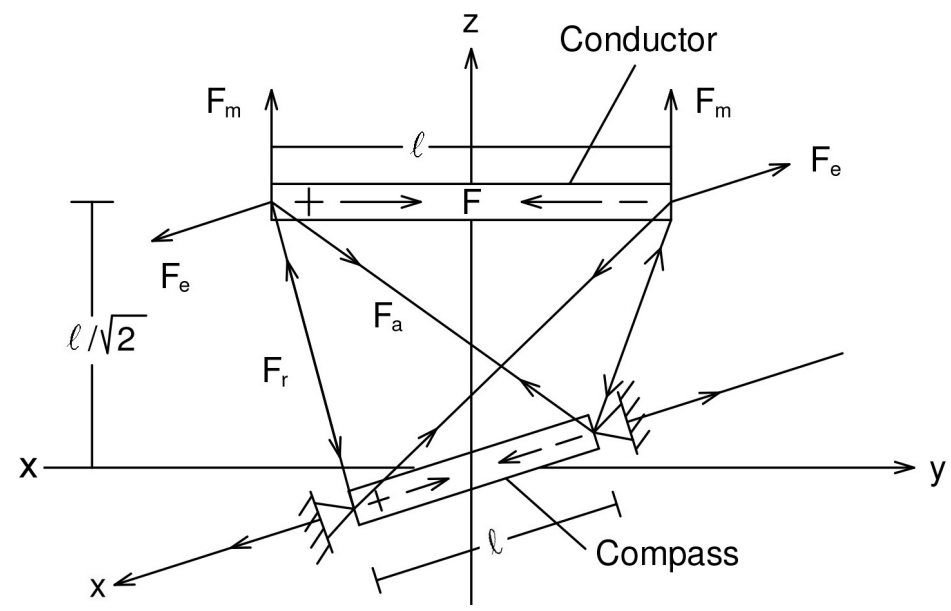

Figure 5. 3D representation of regular tetrahedron formed by dipole-dipole (compass-conductor) interaction.

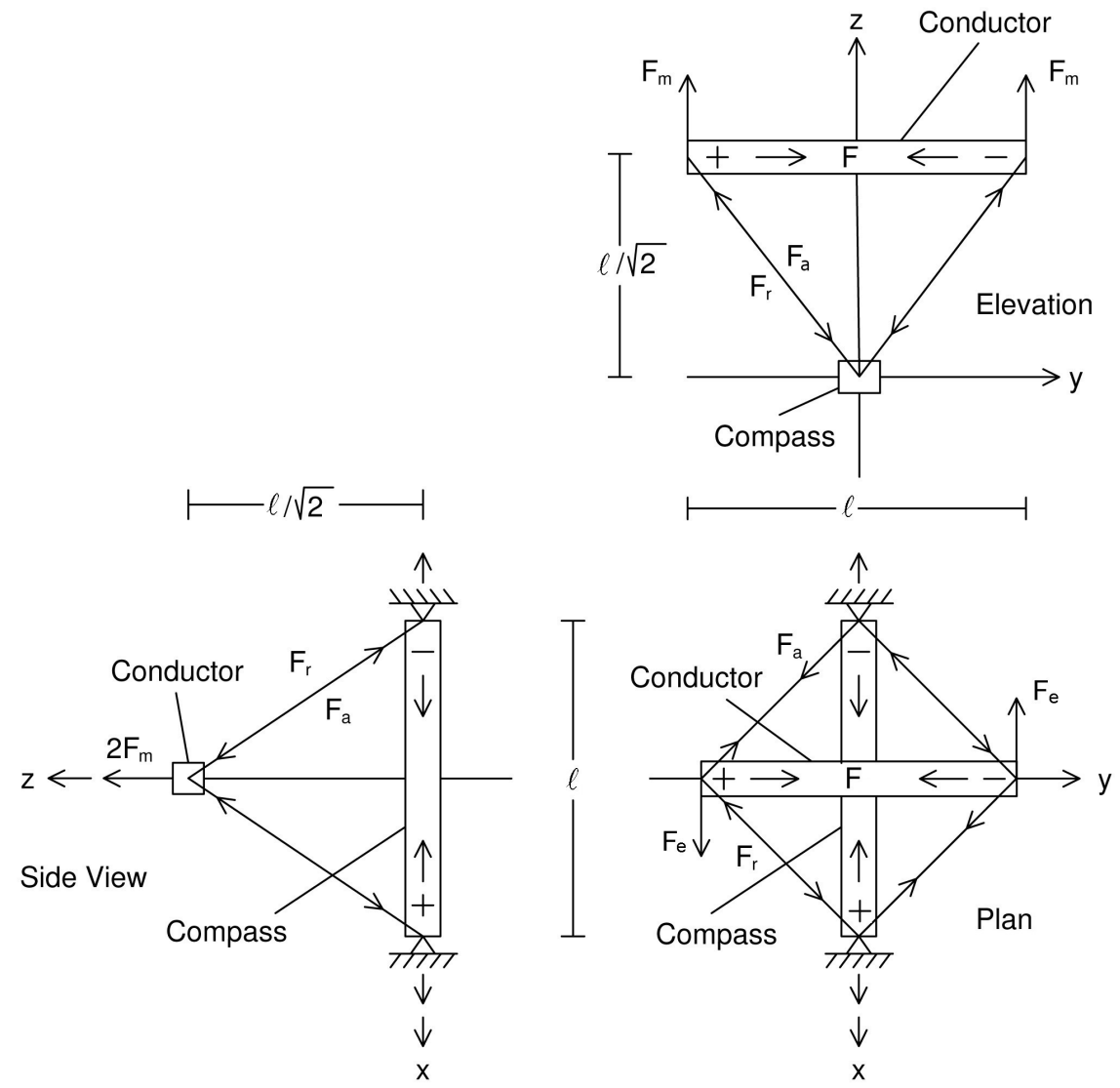

Figure 6. The three projections of regular tetrahedron, formed by dipole-dipole (compass-conductor) interaction: Plan on plane $x y$, Elevation on plane $y z$, Side view on plane $X Z$.

The Compass is fixed, as mentioned previously, in Section 2.1.

Whereas, the Rigid Conductor has 6DOFs (six degrees of freedom), in 3D space. Thus, six corresponding equilibrium conditions have to be written, for the Conductor: Three Forces equations along axes $x, y, z, \sum F_{x}=0, \sum F_{y}=0$, 
$\sum F_{z}=0$, and three Moments equations around axes $x, y, z, \sum M_{x}=0$, $\sum M_{y}=0, \sum M_{z}=0$.

The Forces static equations, along axes $x, y$, as well as, the Moments static equations, around axes $x, y$, are satisfied by symmetry.

Whereas, the Moments static equation, around axis $Z$, is satisfied by a small rotation of Rigid Conductor, around axis $Z$, as will be shown in the numerical example of Section 2.7.

So, the only out-of-balance forces, in the Regular Tetrahedron, acting on Rigid Conductor, are the two forces $F_{m}$, along $Z$, at the ends of Conductor, coinciding with the so-called "magnetic forces", shown in Figure 5 (3D Representation of Regular Tetrahedron), and in Figure 6 (three Projections of regular Tetrahedron), where these two magnetic forces $F_{m}$ are mainly and clearly shown on the Elevation of plane $y z$.

So, the directions and magnitudes of these "magnetic forces" $F_{m}$, along $z$ axis, acting on two ends of Rigid Conductor, are determined, in a simple and Rational way, from Dipole-Dipole (Compass-Conductor) Interaction, with help of a regular Tetrahedron, without recoursing to a "right hand rule", and in agreement with equations based on Experimental Data [1] [2], as will be shown in Numerical Example of Section 2.7 and Applications of Sections 3.1 up to 3.3.

\subsection{Coulomb Repulsion between Like Charges. Lennard-Jones Interaction between Unlike Charges}

In the previous Sections 2.1 up to 2.4, for Attraction between Opposite Electric Charges, within Rigid Compass and Rigid Conductor, the simple Coulomb electrostatic law [11] was used, which works well in these cases.

In the present section, where Dipole-Dipole Interaction, between Compass and Conductor, through free space, is considered, for Repulsion between Like Charges, again the simple Coulomb electrostatic law [11] is taken into account. However, for Interaction (Attraction or Repulsion), between Unlike Electric Charges, a more accurate Lennard-Jones law [12] is used, which gives better results.

So, the adopted here Electrostatic laws, in Dipole-Dipole (Compass-Conductor) Interaction, through free space, are as follows:

1) Between a couple of Like Electric Charges, $q_{1}$ and $q_{2}$, at a distance $r$, a Repulsion occurs, obeying the simple Coulomb Electrostatic law [11], described by Figure $7(\mathrm{a})$, and the equation below:

$$
F=k q_{1} q_{2} / r^{2},
$$

where $k=8.9875 \times 10^{9} \mathrm{Nm}^{2} / \mathrm{C}^{2}$ is the Coulomb electrostatic constant.

2) Between a Couple of Unlike Electric Charges $q_{1}$ and $q_{2}$, at a distance $r$, an Interaction (Attraction or Repulsion) holds, obeying a more accurate Lennard-Jones law [12], described by Figure 7(b) and the equation below

$$
F / F_{0}=-\frac{1}{x^{4}}+\frac{1}{x^{2}},
$$


(a)

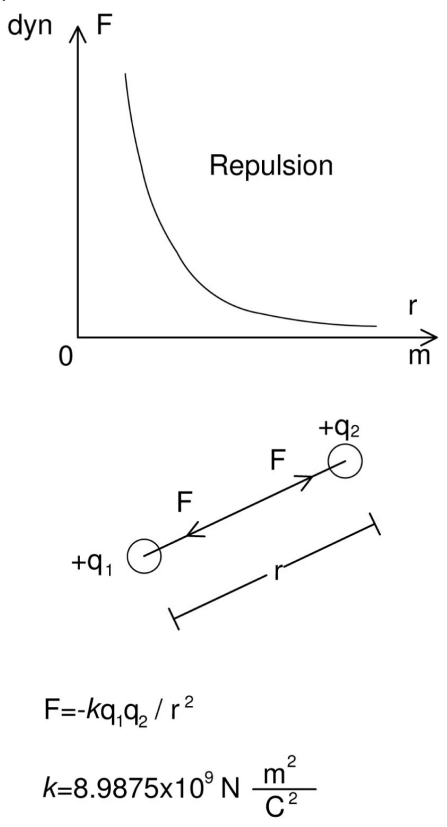

(b)
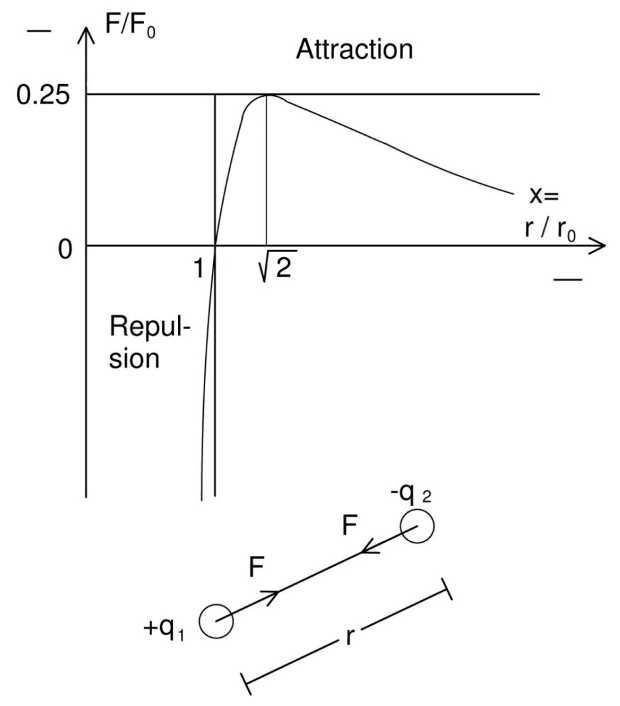

$F / F_{0}=-1 / x^{4}+1 / x^{2}, \quad x=r / r_{0}$

$\mathrm{F}_{0}=+k \mathrm{q}_{1} \mathrm{q}_{2} / \mathrm{r}_{0}^{2}, \quad \mathrm{r}_{0}=\ell / 2$

Figure 7. Electrostatic laws for dipole-dipole (compass-conductor) interaction, through free space. (a) Repulsion between like charges obeying simple Coulomb electrostatic law [11]; (b) Interaction (attraction or repulsion) between unlike charges, obeying a more accurate Lennard-Jones law [12].

where $F_{0}=+k q_{1} q_{2} / r_{0}^{2}, x=r / r_{0}$, and here $r_{0}=\ell / 2$ has been chosen, where $\ell$ side length of Regular Tetrahedron, which gives reasonable results.

\subsection{Numerical Example on Dipole-Dipole (Compass-Conductor) Interaction, with Help of Regular Tetrahedron}

On the basis of previous description of fixed Compass of a constant Electric Field of Section 2.1 and Figure 1, description of Alternating Current in a straight Conductor of Sections 2.2 up to 2.4 and Figures 2-4, and description of Dipole-Dipole (Compass-Conductor) Interaction, with help of Regular Tetrahedron, of Sections 2.5, 2.6 and Figures 5-7, a Numerical Example is shown in present section, as follows.

First, a magnitude of Electric Field

$$
E=200 \mathrm{~N} / \mathrm{C},
$$

typical for Earth Surface, is chosen.

The Electric Charges at the ends of a fixed Compass with length $\ell=1.0 \mathrm{~m}$, are according to Figure 1(b) and Equation (1) of Section 2.1,

$$
\begin{aligned}
q_{1} & =E \ell^{2} / k= \pm 200 \mathrm{~N} / \mathrm{C} \times 1.0^{2} \mathrm{~m}^{2} / 8.9875 \times 10^{9} \mathrm{~N} \cdot \mathrm{m}^{2} / \mathrm{C}^{2} \\
& = \pm 2.225 \times 10^{-8} \mathrm{C}= \pm 0.02225 \mu \mathrm{C}
\end{aligned}
$$

From Figure 4(d) of output of Numerical Experiment of Section 2.4, on Alternating Current in a straight Conductor, the two maximum, in absolute value, opposite charges, at the ends of Conductor are 


$$
q_{2}= \pm 0.45 \times 10^{-6} \mathrm{C}= \pm 0.45 \mu \mathrm{C} .
$$

The maximum attractive force, between two opposite charges, at ends of Conductor, is, according to Figure 4(e).

$$
F=+1.820 \mathrm{mN}=+182.0 \mathrm{dyn} .
$$

The same value can be obtained by Coulomb electrostatic law,

$$
\begin{aligned}
F & =+k q^{2} / \ell^{2}=+8.9875 \times 10^{9} \mathrm{~N} \cdot \mathrm{m}^{2} / \mathrm{C}^{2} \times 0.45^{2} \times 10^{-12} \mathrm{C}^{2} / 1.0^{2} \mathrm{~m}^{2} \\
& =+1.820 \times 10^{-3} \mathrm{~N}=+182.0 \mathrm{dyn}
\end{aligned}
$$

The magnitudes of forces, acting at ends of Conductor, along $x$, due to Electric Field, are according to 3D Representation of Figure 5 and to Plan on plane $x y$ of Figure 6:

$$
F_{e}= \pm E q_{2}= \pm 200 \mathrm{~N} / \mathrm{C} \times 0.45 \times 10^{-6} \mathrm{C}= \pm 9.0 \times 10^{-5} \mathrm{~N}= \pm 9.0 \mathrm{dyn} .
$$

The Repulsion, between Like Electric Charges of Compass and Conductor, is according to Figure 5 and Figure 6 (particularly shown on Plan of $x y$ plane),

$$
\begin{aligned}
F_{r} & =-k q_{1} q_{2} / \ell^{2} \\
& =-8.9875 \times 10^{9} \mathrm{~N} \cdot \mathrm{m}^{2} / \mathrm{C}^{2} \times 0.02225 \times 10^{-6} \mathrm{C} \times 0.45 \times 10^{-6} \mathrm{C} / 1.0^{2} \mathrm{~m}^{2} \\
& =-9.0 \times 10^{-5} \mathrm{~N}=-9.0 \mathrm{dyn}
\end{aligned}
$$

that is, it results with the same magnitude as above $F_{e}$.

The Lennard-Jones Interaction, between Unlike Charges of Compass and Conductor, gives an Attractive force $F_{a}$, according to previous Section 2.6 and Figure 7(b),

$$
\begin{gathered}
r_{0}=\ell / 2=1.0 \mathrm{~m} / 2=0.5 \mathrm{~m}, \quad x=r / r_{0}=1.0 \mathrm{~m} / 0.5 \mathrm{~m}=2 \\
F_{0}=+k q_{1} q_{2} / r_{0}^{2} \\
=+8.9875 \times 10^{9} \mathrm{~N} \cdot \mathrm{m}^{2} / \mathrm{C}^{2} \times 0.02225 \times 10^{-6} \mathrm{C} \times 0.45 \times 10^{-6} \mathrm{C} / 0.5^{2} \mathrm{~m}^{2} \\
=+36 \times 10^{-5} \mathrm{~N}=+36.0 \mathrm{dyn} \\
F_{a}=+F_{0}\left(-\frac{1}{x^{4}}+\frac{1}{x^{2}}\right)=+36 \times 10^{-5} \mathrm{~N} \times\left(-\frac{1}{2^{4}}+\frac{1}{2^{2}}\right) \\
=+36.0 \times 10^{-5} \mathrm{~N} \times 0.1875=+6.75 \times 10^{-5} \mathrm{~N}=+6.75 \mathrm{dyn}
\end{gathered}
$$

All the forces, acting on the Conductor, determined up to now, are depicted in Figure 8(a), in the Plan Projection of Regular Tetrahedron on plane $x y$. In this Figure 8(a), the unit dyn, of all forces, is omitted, for simplicity.

The Compass is fixed in space, as mentioned previously in Section 2.1.

As mentioned in Section 2.5, the static equilibrium equations of Rigid Conductor, $\sum F_{x}=0, \sum F_{y}=0$ and $\sum M_{x}=0, \sum M_{y}=0$ are satisfied by symmetry.

The sum of moments, acting on Rigid Conductor, around axis $z$, is, as shown in Figure 8(a),

$$
\sum M_{z}=[9.0-(9.0+6.75) 0.5] \mathrm{dyn} \times \ell=+1.125 \mathrm{dyn} \times \ell
$$

where 0.5 is direction cosine of sides of Regular Tetrahedron, with respect to $x$ 
(a)

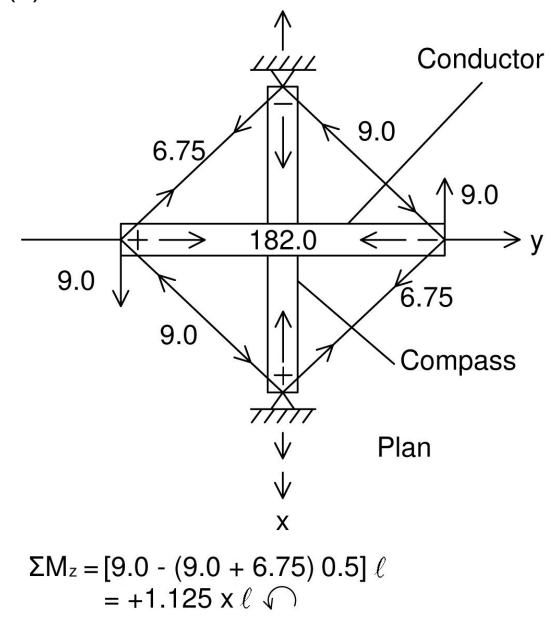

(b)

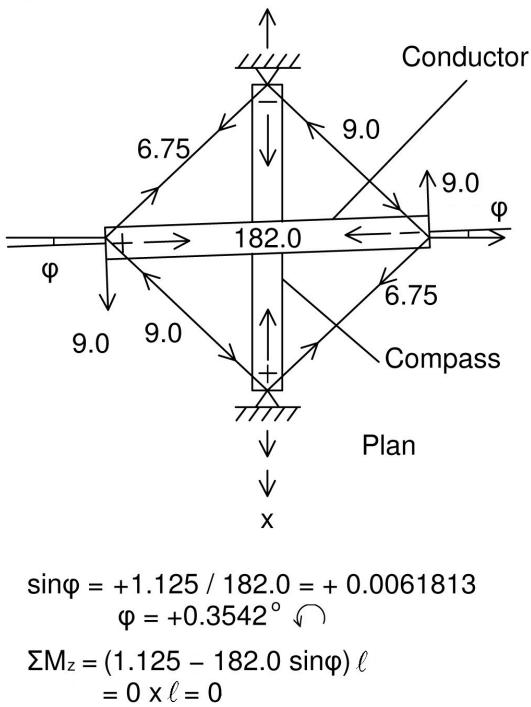

Figure 8. Numerical example on dipole-dipole (compass-conductor) interaction with help of a regular tetrahedron. Plan on $x y$ plane of forces acting on rigid conductor. The unit dyn, of all forces, is omitted, for simplicity. (a) Before small rotation of Rigid Conductor around $z$ axis. (b) After small rotation of Conductor by an angle $\varphi=+0.3542^{\circ}$ around $z$ axis, counter-clock-wise and achievement of a stable equilibrium around $z$ axis.

axis. That is, for the moment, an equilibrium of Rigid Conductor, around $z$ axis, does not exist.

However, the above out-of-balance total moment pushes the Rigid Conductor to rotate counter-clock-wise, around $\mathrm{z}$ axis. When the Rigid Conductor rotates, in this direction, by a small angle $\varphi$ with

$$
\sin \varphi=+1.125 \text { dyn } / 182.0 \text { dyn }=+0.0061813 \text {, }
$$

(where +1.125 dyn is taken from above out-of-balance moment equation and +182.0 dyn is internal attractive force of Conductor), that is, by a small angle $\varphi=+0.3542^{\circ}$ counter-clock-wise, a stable equilibrium around $\mathrm{z}$ axis is achieved, as shown in Figure 8(b)

$$
\sum M_{z}=(+1.125-182.0 \sin \varphi) \mathrm{dyn} \times \ell=0 \times \ell=0 .
$$

And affecting of other forces, from this small rotation of Conductor, is negligible. Now, the only out-of-balance forces, acting on the Rigid Conductor, are the two forces $F_{m}$, along $z$ axis, shown in the Elevation of Regular Tetrahedron, on plane $y z$, of Figure 9:

$$
2 F_{m}=2 \times(9.0-6.75) \text { dyn } \times 0.7071=2 \times 1.591 \text { dyn }=3.182 \text { dyn },
$$

where 0.7071 is the direction cosine of Regular Tetrahedron sides with respect to $z$ axis.

This only out-of-balance force $2 F_{m}=3.182 \mathrm{dyn}$, acting on the Rigid Conductor, along $z$ axis, resulting from Dipole-Dipole (Compass-Conductor) Interaction, in a simple Rational way, with help of a Regular Tetrahedron, is the so-called "magnetic force". 


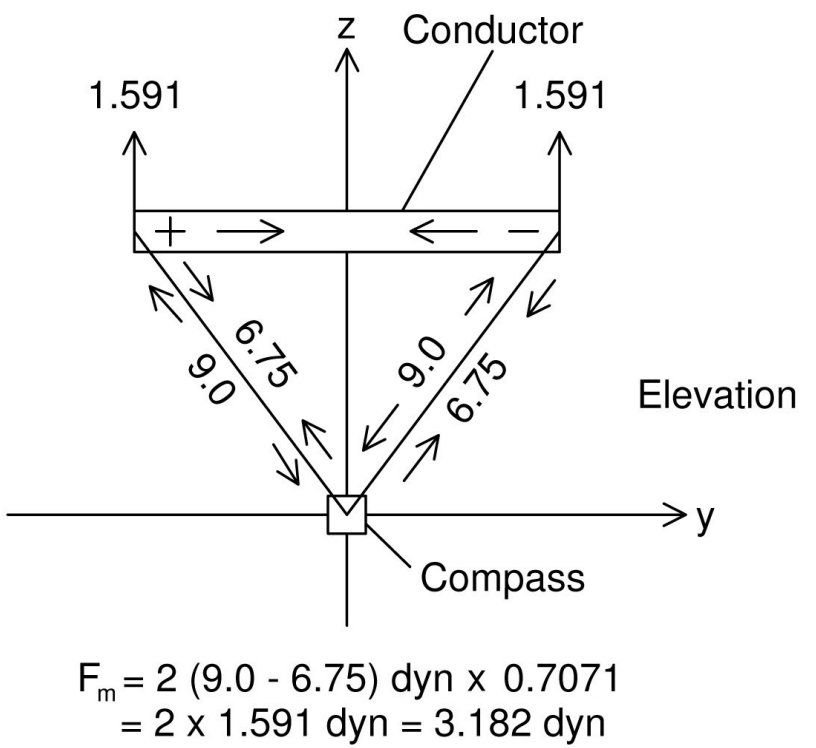

Figure 9. Elevation of regular tetrahedron, that is projection on yz plane, showing the formation of the only two out-of-balance forces $F_{m}$, along $z$ axis, acting on Rigid Conductor, which are the so-called "magnetic forces". The unit dyn, of all forces, is omitted, in figure, for simplicity.

Every physical or numerical experiment exhibits some limitations, due to its simplifications. Main simplifications of present numerical experiment are that Conductor Dipole is perpendicular to Compass Dipole, due to the Regularity of the Tetrahedron used, as well as the simplification resulting from discretization of electric dipoles field.

\section{Applications}

In the following Applications 3.1 and 3.2, the proposed model gives reasonable values for directions and magnitudes of problem variables, when applied to equations of Electro-Magnetism based on Experimental data. Particularly, in the following Application 3.3, results, obtained by the proposed model, are found in close approximation with corresponding ones, obtained by an empirical formula, based on Experimental results by H.-C. Oersted and A.-M. Ampère.

\subsection{Forces Acting on an Electric Charge Moving in an Electric Field}

On the basis of Experimental data, the magnitude of a force $\bar{F}$, acting on an Electric Charge $Q$, moving with a speed $\bar{v}$, in a magnetic field $\bar{B}$, is given by the equation [1] [2]:

$$
F=Q v B \sin \theta,
$$

where $\theta$ is the angle between speed $\bar{v}$ and magnetic field vector $\bar{B}$. Here, for simplicity, $\theta=90^{\circ}$ is assumed, so $\sin \theta=1$, that is $\bar{v}$ is perpendicular to $\bar{B}$, and

$$
F=Q v B
$$


The direction of magnetic field vector $\bar{B}$ is that of a Compass, in this field, and the direction of force $\bar{F}$ is found from $\bar{B}, \bar{v}$ directions, by the first "right hand rule" [1] [2].

In order to apply the proposed here model to the above fundamental Equation (2) of Electro-Magnetism, first, as Electric Charge, is chosen the total Charge of Free Electrons Cloud of numerical example on Alternating current in a straight Conductor, of Section 2.4:

$$
\begin{aligned}
Q & =n A l e \\
& =8.46 \times 10^{28} \text { electrons } / \mathrm{m}^{3} \times 3.0 \times 10^{-6} \mathrm{~m}^{2} \times 1.0 \mathrm{~m} \times 1.6 \times 10^{-19} \mathrm{C} / \text { electron } \\
& =40,608 \mathrm{C}
\end{aligned}
$$

As speed $\mathrm{v}$ of the moving charge, is chosen the maximum absolute drift speed of Free Electrons Cloud, of the same above numerical example of Section 2.4, given in the input, and also shown in Figure 4(b) of output,

$$
\max |v|=2.46257 \times 10^{-4} \mathrm{~m} / \mathrm{sec}=0.246257 \mathrm{~mm} / \mathrm{sec} .
$$

whereas, as maximum absolute magnetic force, is chosen the only out-of-balance force, in Regular Tetrahedron, acting on the Rigid Conductor, from numerical example of Section 2.7, shown in Figure 9:

$$
\max |F|=3.182 \times 10^{-5} \mathrm{~N}=3.182 \text { dyn . }
$$

This value of $\max |F|$ has been obtained by use of the maximum absolute charge, $\max |q|=0.45 \times 10^{-6} \mathrm{C}$ at the ends of Conductor. As shown in Figure 4(b) and Figure 4(d) of Section 2.4, $\max |v|$ and $\max |q|$, thus $\max |F|$, too, do not occur simultaneously, but at two time instants differing by only a fraction of $1.0 \mu \mathrm{sec}=10^{-6} \mathrm{sec}$. It seems that the above fundamental Equation (2) of Electro-Magnetism has also been based on maximum absolute values of $F$ and $V$ occurring at very slightly differing time instants. So, the above values of $Q$, $\max |v|, \max |F|$, of proposed model, are substituted in the fundamental Equation (2) of Electro-Magnetism.

$$
\begin{gathered}
\max |F|=Q \max |v| B \\
3.182 \times 10^{-5} \mathrm{~N}=40,608 \mathrm{C} \times 2.46257 \times 10^{-4} \mathrm{~m} / \mathrm{sec} \times B .
\end{gathered}
$$

From this equation, the magnitude $B$ of the magnetic field is obtained,

$$
\begin{aligned}
B & =3.182 \times 10^{-5} \mathrm{~N} /\left(40,608 \mathrm{C} \times 2.46257 \times 10^{-4} \mathrm{~m} / \mathrm{sec}\right)=3.182 \times 10^{-6} \mathrm{~N} / \mathrm{C} \cdot \mathrm{m} / \mathrm{sec} \\
& =3.182 \times 10^{-6} \mathrm{~N} / \mathrm{A} \cdot \mathrm{m}=3.182 \mu \text { Tesla }
\end{aligned}
$$

which is reasonable.

As regards the position and direction of magnetic field vector $\bar{B}$, these coincide with those of a corresponding Compass of a constant Electric Field, from the numerical example of Dipole-Dipole (Compass-Contactor) Interaction of Section 2.7. And the direction of magnetic force $\bar{F}$ on the moving charge $Q$ results, from the directions of $\bar{B}, \bar{v}$, the same, either determined by the first "right hand rule", based on Experiments [1] [2], or determined by the simple Rational way, of the proposed here Dipole-Dipole (Compass-Contactor) Inte- 
raction, with help of a Regular Tetrahedron.

\subsection{Force on a Current Carrying Straight Conductor Due to a Magnetic Field}

A second fundamental equation of Electro-Magnetism, based on Experimental data, or resulting from the first fundamental Equation (2) of Section 3.1, is that giving the magnitude of force $\bar{F}$ acting on a Current I carrying Conductor, with length $\ell$, due to a magnetic field $\bar{B}$. The magnitudes of the above variables are related to each other, by the equation [1] [2]:

$$
F=B I \ell \sin \theta,
$$

where $\theta$ the angle between directions of Current $I$ and magnetic field vector $\bar{B}$. Here, for simplicity, $\theta=90^{\circ}$ is assumed, thus $\sin \theta=1$, that is, Current $I$ is perpendicular to magnetic field vector $\bar{B}$. So,

$$
F=B I \ell .
$$

By taking into account that $I=n A v e$ and $Q=n A l e$, from Sections 2.3 and 3.1, respectively, it can be shown that the above second fundamental Equation (3) of Electro-Magnetism results from the first one (2) of Section 3.1, as follows:

$$
F=Q v B=n A \ell e v B=n A v e \ell B=I \ell B=B I \ell
$$

In order to apply the proposed model on the above second fundamental Equation (3), the magnitude of magnetic field $B=3.182 \mu \mathrm{T}$ is taken from application 3.1, the length $\ell=1.0 \mathrm{~m}$ of Conductor from numerical examples of Sections 2.4 and 2.7, and maximum absolute Current is obtained from Figure 4(c) or, the same, from input $v_{0}$ of numerical example of Section 2.4:

$$
\begin{aligned}
\max |I|= & n A v_{0} e \\
= & 8.46 \times 10^{28} \text { electrons } / \mathrm{m}^{3} \times 3.0 \times 10^{-6} \mathrm{~m}^{2} \times 2.46257 \times 10^{-4} \mathrm{~m} / \mathrm{sec} \\
& \times 1.6 \times 10^{-19} \mathrm{C} / \text { electron } \\
= & 10.0 \mathrm{C} / \mathrm{sec}=10.0 \mathrm{~A}
\end{aligned}
$$

By substituting the above values of $B, \max |I|, \ell$, in the second fundamental Equation (3), is obtained:

$$
\begin{aligned}
F & =B \max |I| \ell=3.182 \times 10^{-6} \mathrm{~N} / \mathrm{A} \cdot \mathrm{m} \times 10.0 \mathrm{~A} \times 1.0 \mathrm{~m} \\
& =3.182 \times 10^{-5} \mathrm{~N}=3.182 \mathrm{dyn}
\end{aligned}
$$

which coincides with the out-of-balance force on the Rigid Conductor, resulting in the numerical example of Section 2.7, from Dipole-Dipole (Compass-Conductor) Interaction, with help of a Regular Tetrahedron, as was expected.

\subsection{Comparison of Proposed Model with Oersted-Ampére Rule}

Hans-Christian Oersted [13] and André-Marie Ampère [14] observed that, near and around a straight Conductor, with zero current, compasses are parallel to Earth Surface Magnetic Field, as shown in Figure 10(a). However, if the Conductor carries a Current $I$, the compasses change their directions and are arranged, on planes perpendicular to Conductor, tangentially to circles, with centers 

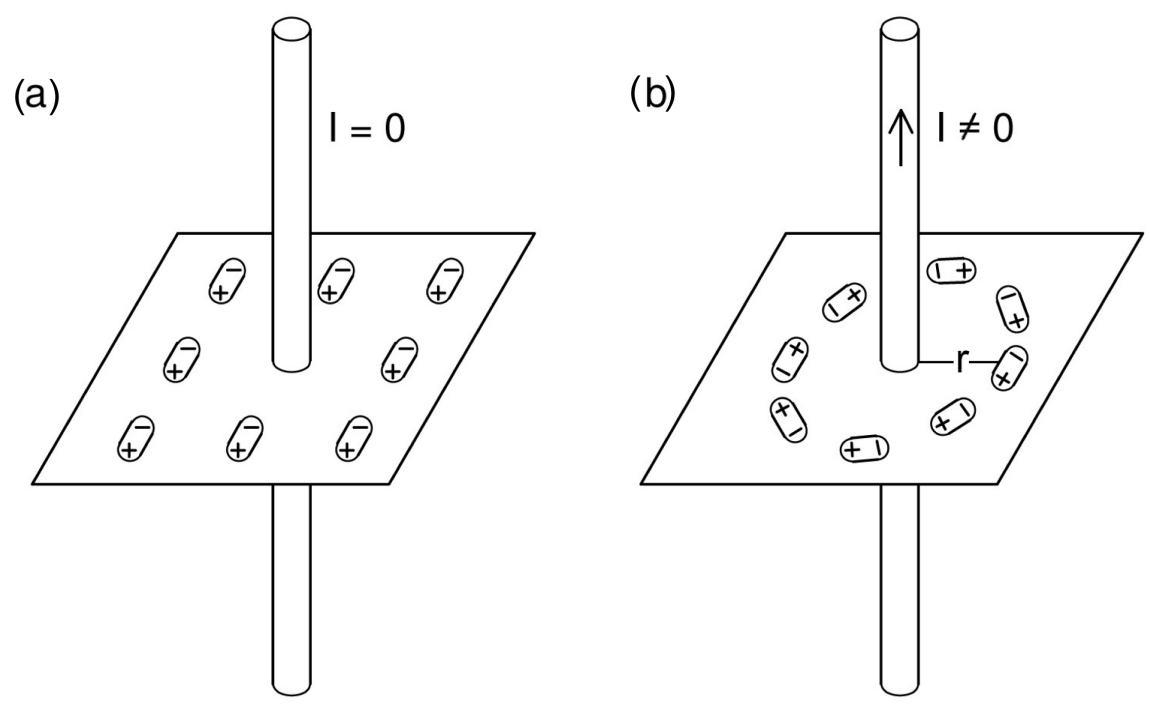

Figure 10. (a) For zero current $I=0$, in the Conductor, the compasses are parallel to Earth surface magnetic field. (b) For nonzero Current $I \neq 0$, in the straight Conductor, the compasses are arranged, on planes perpendicular to Conductor, tangentially to cycles, with centers on the Conductor.

on the Conductor, as shown in Figure 10(b). These compasses show that magnetic fields are created around the Current carrying Conductor. An empirical formula [1] [2] estimates the magnitudes B of these magnetic fields:

$$
B=\mu_{0} I / 2 \pi r,
$$

where the constant $\mu_{0}=4 \pi \times 10^{-7} \mathrm{~T} \cdot \mathrm{m} / \mathrm{A}$ is called permeability of free space, $I$ is the Current carried by the straight Conductor and $r$ is the distance between Compass and Conductor.

This empirical formula works well and gives weak magnetic fields around a Current carrying straight Conductor, except in the case that $r \rightarrow 0$ implying $B \rightarrow \infty$, which is non-realistic.

In order to apply the proposed model to the above empirical formula (4), the compass-conductor distance $r=\ell / \sqrt{2}=1.0 \mathrm{~m} / \sqrt{2}=0.7071 \mathrm{~m}$, from example of Section 2.7, and the maximum absolute current $I=\max |I|=10.0 \mathrm{~A}$, from example of Section 2.4, are substituted in the empirical formula (4) and is obtained:

$$
B=\frac{\left(4 \pi \times 10^{-7} \mathrm{~T} \cdot \mathrm{m} / \mathrm{A}\right) \times 10.0 \mathrm{~A}}{2 \pi \times 0.7071 \mathrm{~m}}=2.828 \times 10^{-6} \mathrm{Tesla}=2.828 \mu \mathrm{T}
$$

whereas, the proposed model, for the same Current $I=10.0$ A and Compass-Conductor distance $r=0.7071 \mathrm{~m}$, has given, in the application of Section 3.1, a magnetic field $B=3.182 \times 10^{-6} \mathrm{~T}=3.182 \mu \mathrm{T}$.

The value of magnetic field $B=2.828 \mu$ Tesla , obtained by the above empirical formula (4), is in satisfactory approximation with the corresponding value $B=3.182 \mu$ Tesla, obtained by the proposed model. So, the reliability of the proposed model is checked. 


\section{Conclusions}

1) Any Electric Field can produce a Field of many small, continuously distributed in space, Electric Dipoles. In a region where the Electric Field is constant, in direction and magnitude, all the small Dipoles are parallel to the Electric Field and are represented by a single, long, fixed in space, parallel to them, Electric Dipole, which is here called Compass.

2) An Alternating Current, in a straight Conductor, is studied by a simple and short computer program, for step-by-step nonlinear dynamic analysis. It is found that only an Alternating current, not a direct current, can produce an Electric Dipole in the straight Conductor.

3) The above two Electric Dipoles, the fixed Compass of a constant Electric Field, and the Alternating current carrying straight Conductor, are assumed with equal lengths $\ell$, lying on two skew lines, perpendicular to each other, at a distance $\ell / \sqrt{2}$, thus forming by their four ends, a Regular Tetrahedron with side length $\ell$.

4) In the Compass-Conductor Interaction, the Repulsion, between a couple of Like Electric Charges, obeys the simple Coulomb electrostatic law. Whereas, the Interaction (Attraction or Repulsion), between a couple of Unlike Electric Charges, obeys a more accurate Lennard-Jones law.

5) The analysis of Dipole-Dipole (Compass-Conductor) Interaction, with help of a Regular Tetrahedron, can be performed by a hand calculator. It is found that the only out-of-balance forces, in the Regular Tetrahedron, acting on the rigid Conductor, are the so-called "magnetic forces". Their direction is found, in a simple Rational way, with help of a Regular Tetrahedron, without recoursing to a "right hand rule".

6) The proposed model is applied to a. The force acting on an Electric Charge moving in a magnetic field. $b$. The force acting on a current carrying straight Conductor, due to a magnetic field. c. The magnetic fields created around a current carrying straight Conductor, and gives reasonable results in these cases.

7) Particularly, in the third application, results, obtained by the proposed model, are found in satisfactory approximation with corresponding ones, obtained by an empirical formula, based on relevant Experimental observations by H.-C. Oersted and A.-M. Ampère. So, the reliability of proposed model is checked.

8) The position and direction of a magnetic field vector coincide with those of a corresponding fixed Compass, in a region of Constant Electric Field. Whereas, the magnitude of the magnetic field vector can be obtained by dividing the out-of-balance magnetic force on an Alternating current carrying Conductor by the current and length of the Conductor.

9) Main finding of present work is that, without introducing the concept of a magnetic field vector, by combining the dipoles field produced by an electric field, with the dipole of an alternating current carrying conductor, the magnetic forces can be determined. 
10) Even in a direct current, small oscillations of free electrons exist, which create small alternating currents, thus small electric dipoles. In future research, the significance of these small oscillations can be investigated.

\section{Conflicts of Interest}

The authors declare no conflicts of interest regarding the publication of this paper.

\section{References}

[1] Serway, R.A. and Vuille, C. (2012) College Physics. 9th Edition, Brooks/Cole Cengage Learning.

[2] Hayt Jr, W.H. and Buck, J.A. (2001) Engineering Electro-Magnetics. Series in Electrical and Computer Engineering. 6th Edition, McGraw-Hill, USA.

[3] Argyris, J.H. (1965) Continua and Discontinua; An Apercu of Recent Developments of the Matrix Displacement Method. Air Force Conference on Matrix Methods in Structural Mechanics, Wright Patterson Air Force Base, Dayton, Ohio, 26-28 October 1965.

[4] International Conferences FeNoMech (Finite Elements in Nonlinear Mechanics). ISD (Institut für Statik und Dynamik). University of Stuttgart, Germany. I. $30 \mathrm{Au}$ gust-1 September 1 1978, II 25-28 August 1981. III. 10-14 September 1984. Proceedings in the Journal CMAME (Computer Methods in Applied Mechanics and Engineering).

[5] Newmark, N.M. (1959) A Method of Computation in Structural Dynamics. Journal of Engineering Mechanics ASCE, 85, 67-94.

[6] Argyris, J.H. and Scharpf, D.W. (1969) Finite Elements in Time and Space. Nuclear Engineering and Design, 10, 456-464.

https://doi.org/10.1016/0029-5493(69)90081-8

[7] Papadopoulos, P.G. (1984) A Simple Algorithm for the Nonlinear Dynamic Analysis of Networks. Computers and Structures, 18, 1-8.

https://doi.org/10.1016/0045-7949(84)90074-9

[8] Wikipedia (2019) Michael Faraday.

[9] Wikipedia (2019) Nikola Tesla.

[10] Wikipedia (2019) Tetrahedron.

[11] Wikipedia (2019) Charles-Augustin de Coulomb.

[12] Wikipedia (2019) Lennard-Jones Potential.

[13] Wikipedia (2019) Hans-Christian Oersted.

[14] Wikipedia (2019) André-Marie Ampère. 